\title{
La facturation électronique selon le TARMED
}

\author{
Dr Jacques de Haller ${ }^{a}$, Dr A. Haefeli ${ }^{b}$, Dr F. Muggli ${ }^{c}$
}

Quinze mois après l'entrée en vigueur du TARMED, 80\% des médecins libres praticiens établissent déjà leurs factures de manière électronique, remplissant ainsi les dispositions contractuelles bien avant le $1^{\text {er }}$ janvier 2006. Néanmoins, la facturation électronique est sujette à de nombreux malentendus, elle génère des questions et malheureusement aussi des incertitudes. Nous présentons ci-dessous la position de la FMH et des sociétés cantonales de médecine à ce sujet.

\footnotetext{
a Président de la FMH

b Président du G7

c Responsable du groupe de travail TARMED FMH
}

\section{Conditions générales en vertu de la LAMal et du TARMED}

La notion de facturation électronique est apparue avec le tarif uniforme TARMED. Elle figure aussi bien dans la LAMal que dans le tarif luimême, mais elle est malheureusement souvent mal interprétée.

\section{Art 42, $3^{e}$ al. LAMal}

Cet article stipule que le fournisseur de prestations doit remettre au débiteur de la rémunération une facture détaillée et compréhensible. Le fournisseur de prestations est donc responsable de l'établissement de la facture et de son envoi au débiteur.

\section{TARMED}

Dans les interprétations générales (IG-50), le TARMED définit la facturation électronique de la manière suivante: «le critère de facturation électronique est rempli lorsque la facture est transmise à l'assureur par voie électronique, selon la norme fixée par les partenaires contractuels concernés.»

Dans la prestation technique, le TARMED prévoit une indemnisation pour les frais de personnel, d'exploitation et d'infrastructure. La facturation électronique, pour sa part, ne bénéficie d'aucune position spécifique.

\section{Commentaires de la FMH et des sociétés cantonales de médecine (G7)}

a) L'interprétation générale de la facturation électronique concerne spécifiquement le système du tiers payant. Dans le système du tiers garant, le fournisseur de prestations remet sa facture directement au patient. L'IG 50 ne peut pas imposer aux cantons appliquant le système du tiers garant (facture au patient) de passer à celui du tiers payant (facture à l'assureur) car ce changement

\section{Résumé}

Grâce à une procédure spécialement mise au point pour le système du tiers garant, les assureurs peuvent, après réception de la facture envoyée par l'assuré, en obtenir une copie électronique auprès de l'un des centres fiduciaires (TrustCenter) créés à cet effet. Ce mode de faire entièrement automatisé leur permet d'économiser du temps et de l'argent dans la saisie et le contrôle des factures. Les données étant désormais transférées du cabinet médical à l'assureur-maladie sans interruption technique, leur contenu est d'une qualité irréprochable. Pour les assureurs, ce service est une plus-value véritable car il leur permet d'optimiser le traitement administratif et le contrôle des factures, coûteux en termes de ressources humaines. Les économies ainsi réalisées sont substantielles.

nécessite, en vertu de la LAMal, une convention bilatérale entre les parties.

b) La compétence décisionnelle concernant le mode de facturation se trouve chez les partenaires tarifaires. Lorsqu'ils ne peuvent pas s'entendre, la LAMal stipule le système du tiers garant comme norme. Les conventions d'adhésion au TARMED actuellement en vigueur, approuvées par les autorités cantonales, doivent donc être respectées.

c) Compte tenu de ces conventions cantonales, un médecin ne peut passer, à titre individuel, du système du tiers garant à celui du tiers payant qu'en cas de résiliation ou de modification des conventions. Toute autre affirmation à ce sujet est trompeuse, aussi bien pour les fournisseurs de prestations que pour les patients.

\section{Devoirs du médecin dans le système du tiers garant}

En vertu de la convention-cadre TARMED et des conventions cantonales d'adhésion, le médecin qui envoie sa facture directement à ses patients doit tenir une copie électronique de celle-ci à la disposition de l'assureur. 
Art. 11 de la Convention-cadre: Facturation et rémunération

L'annexe 3 à la Convention-cadre précise ceci au chiffre 2 Facturation:

«Les parties contractantes s'engagent à appliquer les dispositions concernant le formulaire de facturation unifié, la facturation électronique et le transfert de données médicales conformément à l'article $11,4^{\mathrm{e}}, 5^{\mathrm{e}}$ et $6^{\mathrm{e}}$ al., ainsi qu'à l'article 14, $2^{\mathrm{e}}$ al., de la conventioncadre TARMED.

L'application, tant sur le plan technique que sur celui du contenu, et la réglementation concernant la charge des frais se fondent sur les normes et les directives élaborées dans le cadre du ‘Forum pour l'échange électronique des informations et des données dans le domaine de la santé> [...].»

La question de savoir comment les données doivent être préparées et transmises en vertu de l'annexe 3 n'a pas encore fait l'objet d'une réglementation jusqu'à ce jour.

\section{Commentaires de la FMH et des sociétés cantonales de médecine (G7)}

a) Environ $80 \%$ des médecins libres praticiens établissent aujourd'hui leurs factures de manière électronique; elles sont donc à disposition au cabinet.

b) Plus de $60 \%$ des médecins libres praticiens ont opté pour un intermédiaire (MediData, TrustCenter, etc.), lequel rassemble les copies électroniques des factures, les garde en sécurité et se charge de les mettre à disposition des assureurs par le biais d'une solution internet centralisée et répondant aux normes de la protection des données.

c) En automne 2003, la délégation commune de négociation de la FMH/G7 et de santésuisse a élaboré une convention réglant les modalités de l'échange électronique des données de facturation. Cette convention a été approuvée par les sociétés cantonales de médecine, mais rejetée par santésuisse qui n'acceptait pas la solution proposée.

d) Ce rejet sans contre-projet concret a contraint certains assureurs-maladie et intermédiaires à résoudre eux-même la question de l'échange des données. Depuis lors, de nouvelles voies ont été testées et mises en œuvre avec succès. Aujourd'hui, l'échange électronique de données de facturation est utilisé dans les faits et a fait ses preuves dans les cabinets médicaux.
En étant $80 \%$ à pratiquer la facturation électronique, les médecins en pratique privée ont accompli leur devoir en la matière. Ils remplissent ainsi les conditions posées pour la facturation électronique et celles permettant de supprimer les limitations. En créant des centres fiduciaires (TrustCenter), ils ont de surcroît volontairement et à leurs frais - élaboré les outils nécessaires à l'obtention des données par les assureurs-maladie.

La FMH et le G7 sont d'avis que tous les médecins tenant leurs données à disposition dans un centre fiduciaire ou chez un autre intermédiaire remplissent largement les conditions posées pour la facturation électronique. Il y a donc lieu de supprimer les limitations dans leur cas.

\section{Indemnisation des prestations fournies par des intermédiaires}

Dans une lettre datée du 8 février 2005, le Dr H. H. Brunner, vice-directeur de l'OFSP, relève ce qui suit:

- Aucune taxe ne peut être perçue pour la facturation. Le fournisseur de prestations doit remettre sa facture au débiteur sans demander d'indemnisation supplémentaire.

- Les assureurs-maladie sont libres de conclure des conventions pour rémunérer des prestations supplémentaires véritables (fournies p.ex. par des intermédiaires) permettant la consultation des factures établies selon le TARMED, aux fins d'économiser des frais administratifs ou d'optimiser le contrôle des factures.

\section{Commentaires de la FMH et des sociétés cantonales de médecine (G7)}

a) Sous la direction de la firme NewIndex, le corps médical a érigé onze centres fiduciaires dans toute la Suisse, lesquels collectent aujourd'hui déjà des factures TARMED établies par plus de sept mille cabinets médicaux, les centralisent et les mettent à la disposition des assureurs-maladie. Ceux-ci peuvent ainsi s'épargner la collecte et le regroupement des données de nombreux cabinets. On estime que les assureurs-maladie pourraient économiser annuellement plus de 20 millions de francs s'ils utilisaient les services de ces intermédiaires.

b) Lors de la mise en service des centres fiduciaires, une attention particulière a été 
accordée aux dispositions légales relatives au secret médical et à la protection des données: les données sont pseudonymisées et codées, au cabinet médical déjà, en vue d'être transférées par internet. Les assureurs profitent ainsi du savoir-faire des centres fiduciaires et du soin apporté à l'application technique de la transmission des données de facturation, auxquelles ils peuvent accéder en toute sécurité et sans investissement propre.

c) Les assureurs sont malheureusement peu nombreux à collecter par voie électronique les factures établies selon le TARMED. Ils n'utilisent donc pas les moyens mis à leur disposition pour économiser des frais administratifs.

- Les médecins libres praticiens sont en mesure de remettre des factures détaillées à leurs débiteurs (généralement les patients).
- La plupart des médecins sont en mesure d'établir correctement les copies électroniques de factures au moyen du logiciel installé dans leur cabinet et de les transmettre aux centres fiduciaires où les assureurs peuvent se les procurer. Cette façon de préparer, archiver et transmettre des données complètes, fiable en tous points selon les tests effectués et conforme à la protection des données, est un outil très utile que nous mettons à la disposition des assureurs. La saisie, le traitement administratif et la plausibilisation des factures s'en trouvent en effet grandement simplifiés!

Nous demandons aux assureurs de reconnaître les avantages procurés par ce mode de faire et de tirer parti des économies pouvant être réalisées dans ce contexte. 